\title{
Fibromixoma Odontogénico Agresivo en Maxila: Abordaje Quirúrgico
}

\author{
Aggressive Odontogenic Fibromixoma in Maxilla: Surgical Approach
}

Gerardo José Guillen Rivera1; Ana Isabel Ortega-Pertuz² \& Michelle Leinin Espina Suarez ${ }^{3}$

GUILLEN, R. G. J.; ORTEGA-PERTUZ, A. I. \& ESPINA, S. M. L. Fibromixoma odontogénico agresivo en maxila: abordaje quirúrgico. Int. J. Odontostomat., 13(1):58-63, 2019.

RESUMEN: El fibromixoma odontogénico (FM) es una infrecuente lesión benigna que muestra un comportamiento invasivo. Considerada una variante del mixoma odontogénico, presenta un origen mesenquimal controvertido y ligera predilección por el sexo femenino. La lesión está asociada a zonas con estructuras dentales adyacentes, siendo su localización más frecuente la región mandibular posterior. El objetivo de este trabajo fue mostrar un caso agresivo de FM en una paciente, localizado en la zona posterior de maxila, destacando su abordaje quirúrgico. Se reporta un caso de una mujer de 52 años, la cual acudió a la consulta por presentar una masa indolora, firme a la palpación en el lado derecho de la maxila. Radiográficamente se observaba como una imagen radiopaca, de márgenes mal definidos que se extendía en el seno maxilar ipsilateral; la tomografía computarizada pudo evidenciar la expansión de las paredes del antro y la fosa nasal, con compromiso del cigomático y el piso de órbita. Se realizó biopsia incisional y el diagnóstico histopatológico fue de FM. El tratamiento de elección consistió en la resección en bloque,mediante abordaje Weber-Fergusson, acompañada de reconstrucción con placa y malla de titanio para garantizar la suspensión del globo ocular; un nuevo estudio histopatológico, confirmó el diagnóstico inicial. Al año del procedimiento quirúrgico no se evidenció recidiva, observando que el material de reconstrucción mantiene su posición, proporcionado estética y funcionabilidad a la paciente.

PALABRAS CLAVE: tumor odontogénico, mixoma, fibromixoma, maxila.

\section{INTRODUCCIÓN}

El fibromixoma odontogénico (FM) es una rara lesión de naturaleza benigna, localmente invasiva y agresiva, de posible origen mesenquimal que aparece en el esqueleto facial, afectando por lo general la zona posterior de la mandíbula en individuos entre la segunda y tercera décadas de la vida (Dietrich et al., 2011; Meleti et al., 2015), con leve predilección por el género femenino. Aunque clínicamente suele ser asintomático (Infante-Cossío et al., 2011; Reddy et al., 2013), puede cursar con dolor y deformidad facial (Dietrich et al.; Cordero Carrasco et al., 2013; InfanteCossío et al.). La lesión es considerada una variante del mixoma odontogénico, conteniendo una cantidad considerable de fibras colágenas dispersas en el estroma mixoide (Meleti et al.; Omeje et al., 2015).
La imagen radiográfica del FM puede ser uni o multilocular, con características expansivas, de márgenes corticalizados cuando se presenta en mandíbula, mientras que en la maxila suelen ser mal definidos, causa desplazamiento de los dientes adyacentes, así como reabsorción radicular externa de los mismos (Infante-Cossío et al.; Gupta et al., 2010; Gandhiraj et al., 2013; Meleti et al.; Omeje et al.).

El tratamiento del FM varía desde la implementación de técnicas conservadoras de enucleación y curetaje con osteotomía periférica (Dietrich et al.; Reddy et al.; Zayet et al., 2014; Kant et al., 2015); acompañado de fijación química (Gandhiraj et al.), a la resección en bloque (Infante-Cossío et al.; Gupta et

\footnotetext{
${ }^{1}$ Cirujano Oral y Maxilofacial. Doctor en Odontología. Guillen-Rivera Oral and Maxillofacial Surgery PLLC. El Paso, Texas, Estados Unidos de América.

2 Doctora en Odontología. Instituto de Investigaciones, Facultad de Odontología, Universidad del Zulia, Maracaibo, estado Zulia, Venezuela.

${ }^{3}$ Doctora en Odontología. El Paso Family Orthodontics. El Paso, Texas, Estados Unidos de América.
} 
al.; Kumar et al., 2012). La elección de una u otra terapéutica está relacionada con el tamaño de la lesión, el compromiso de las estructuras vecinas, así como la posibilidad de la reconstrucción (Cordero Carrasco et al.; Gupta et al.; Kant et al.; Kumar et al.; Zayet et al.). Los FM muestran un porcentaje de recidiva entre el 23 al $45 \%$, teniendo un pronóstico menos favorable en los maxilares que cuando se localiza en otros huesos (Dietrich et al.).

En este trabajo se presenta un caso clínico de FM en maxila, en una paciente de género femenino de 52 años de edad, que comprometió el piso de la órbita, con énfasis en el abordaje quirúrgico.

\section{RELATO DEL CASO}

Paciente de género femenino de 52 años quien acudió al Servicio de Cirugía Bucal y Maxilofacial del Hospital Universitario de Maracaibo, estado Zulia, por presentar un aumento de volumen en el tercio medio facial de cinco años de evolución, que producía gingivorragia, dificultad para hablar y halitosis. Al interrogatorio no manifestó ningún antecedente médico personal y/o familiar de importancia o que guardara relación con el motivo de consulta, considerada de esta manera ASA I.

Al examen físico extraoral se observó asimetría facial del lado derecho, surco nasogeniano borrado, disminución del tamaño ocular y la ventana nasal ipsilateral (Fig. 1A,B). En el examen intraoral se evidenció una lesión expansiva, localizada en el lado derecho de la maxila, que se extendía en su cara vestibular y el paladar duro (Fig. 2), de consistencia firme, que involucraba los dientes 1.4 y 1.5 , con presencia de úlcera debido a trauma oclusal, sin parestesia o limitación de la abertura oral, la mucosa mostraba un color rosa intenso.
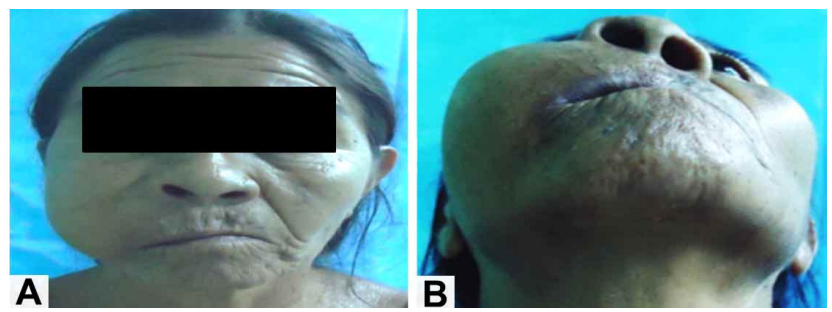

Fig. 1. A. Vista frontal de la paciente donde se observa aumento de volumen del tercio medio de la cara. B. Vista inferior evidenciando alteración del ángulo nasogeniano y ventana nasal disminuida.

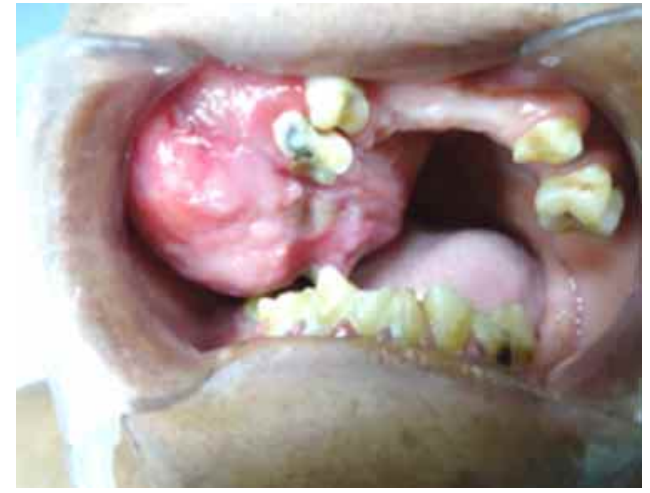

Fig. 2. Vista introral evidenciando lesión expansiva en el lado derecho de la maxila.

La ortopantomografía evidenció una lesión radiopaca, unilocular, de límites difusos, que se extendía desde la línea media hasta la zona posterior del maxilar derecho, invadiendo el seno maxilar respectivo (Fig. 3). En la tomografía computarizada (TC) se observó la expansión de las paredes medial, anterior y posterolateral del antro (Fig. 4A,B), con comprometimiento de la región orbitaria y el hueso cigomático.

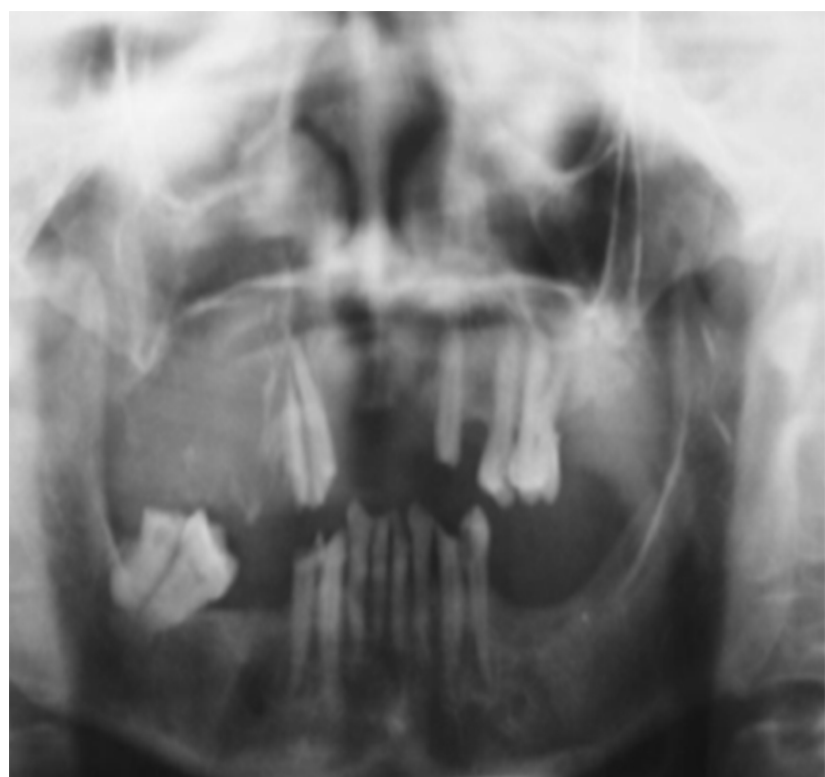

Fig. 3. Ortopantomografía mostrando lesión radiopaca expansiva de márgenes difusos en seno maxilar derecho.

El estudio histopatológico (después de la biopsia incisional) reportó la presencia de tejido conectivo fibrolaxo, mixoide en casi toda su extensión, constituido por fibroblastos redondos, fusiformes y angulados. Se identificaron islas epiteliales consideradas restos odontogénicos, además de vasos sanguíneos de cali- 

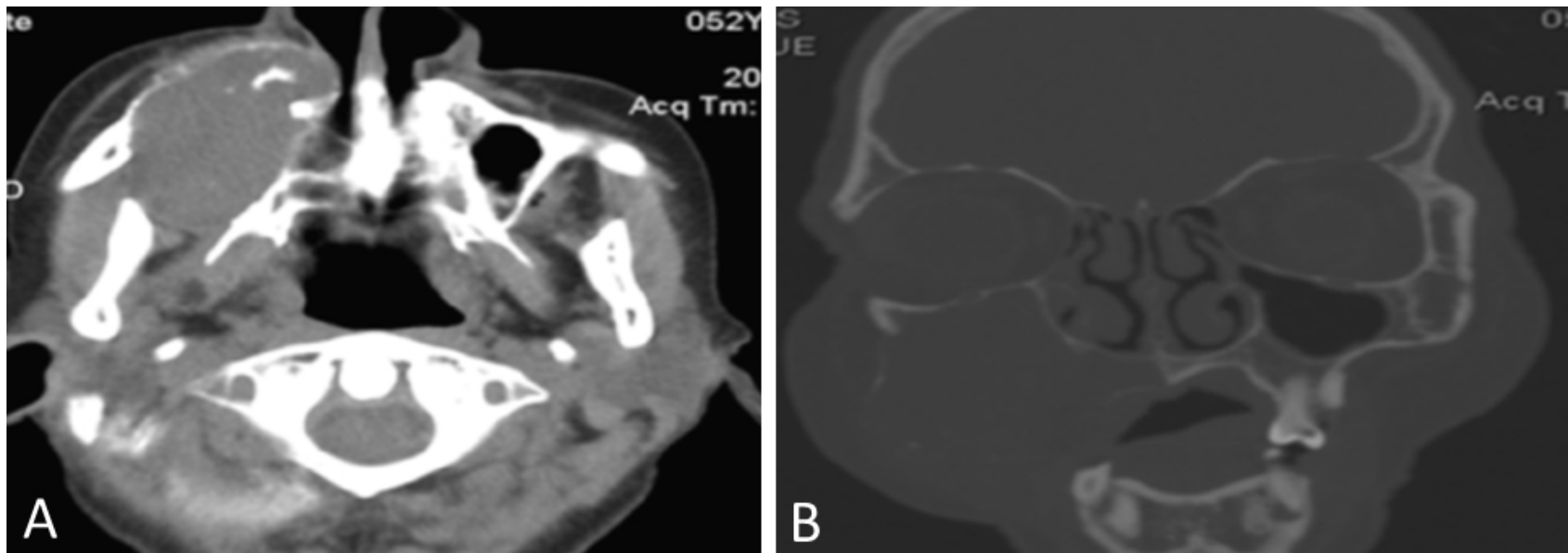

Fig. 4. A. Vista axial y B. Vista coronal de tomografía computarizada en las que se observa expansión de las paredes medial, anterior y posterolateral del seno maxilar derecho.
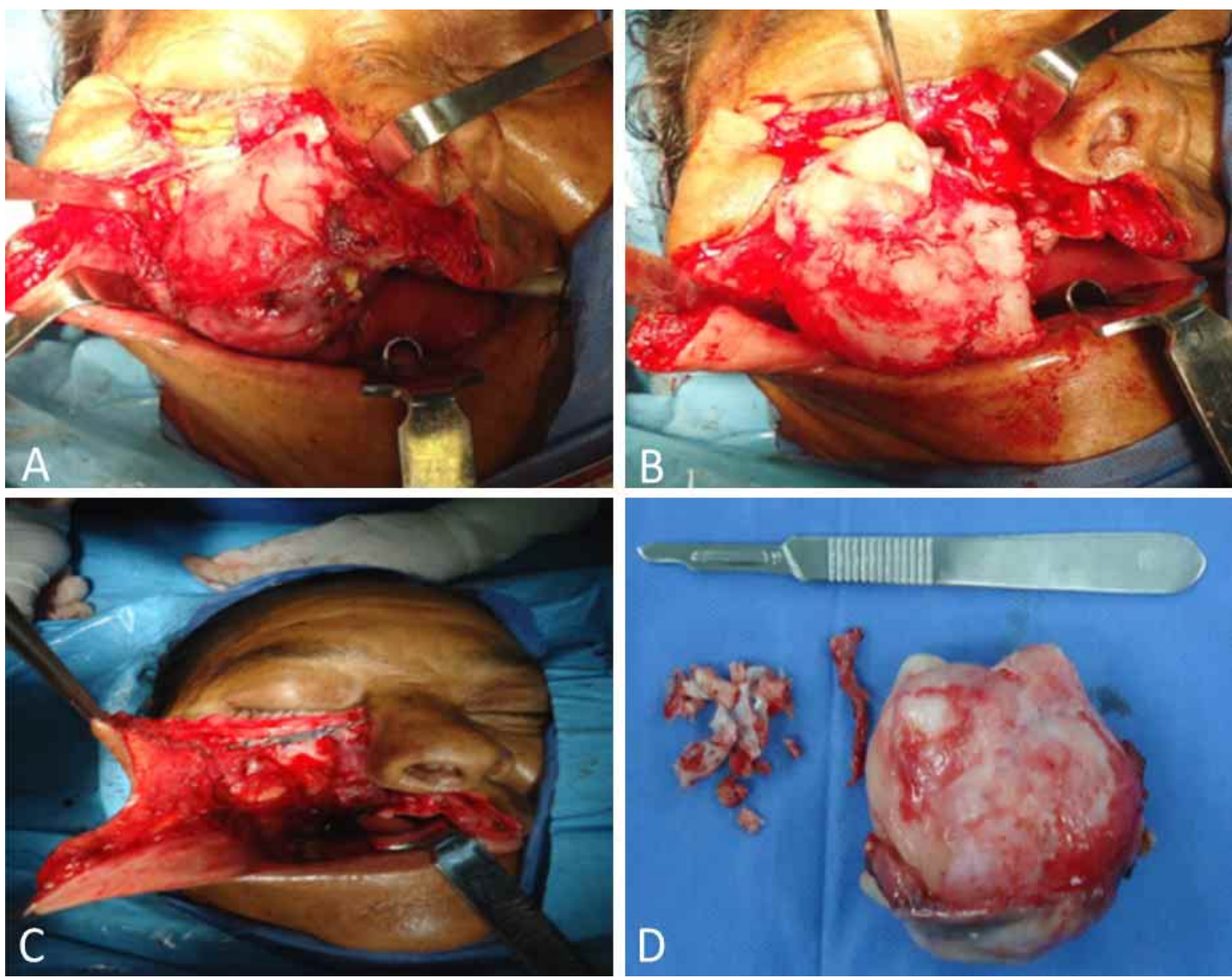

Fig. 5. A. Abordaje extraoral Weber-Ferguson e intraoral en mucosa del surco vestibular. B. Placa de reconstrucción de 1,5 adaptada mediante tornillos monocorticales. C.Acto Quirúrgico. D. Aspecto macroscópico del espécimen quirúrgico. 
bre variado, dilatados y un leve infiltrado inflamatorio mononuclear. El diagnóstico definitivo de la lesión fue de FM.

Abordaje quirúrgico. Una vez considerado el diagnostico histopatológico, la naturaleza infiltrativa de la lesión y su localización, la paciente fue tratada con hemimaxilectomia mediante un abordaje extraoral Weber-Fergusson e intraoral en mucosa del surco vestibular y en línea media del paladar duro, bajo anestesia general balanceada e intubación orotraqueal siguiendo la normas universales de asepsia y antisepsia.

Se realizó la demarcación de las líneas de incisión con azul de metileno y se aplicó anestesia infiltrativa con lidocaína $1 \%$ con epinefrina 1:100.000 $(10 \mathrm{ml})$ aplicada intra y extraoralmente con fines hemostáticos. La hemimaxilectomia se extendió desde la línea media del maxilar derecho con resección nasal media, seno maxilar, piso de órbita, margen infraorbitario y parte del hueso zigomático (Fig. 5A). Los órganos dentarios asociados a la lesión fueron

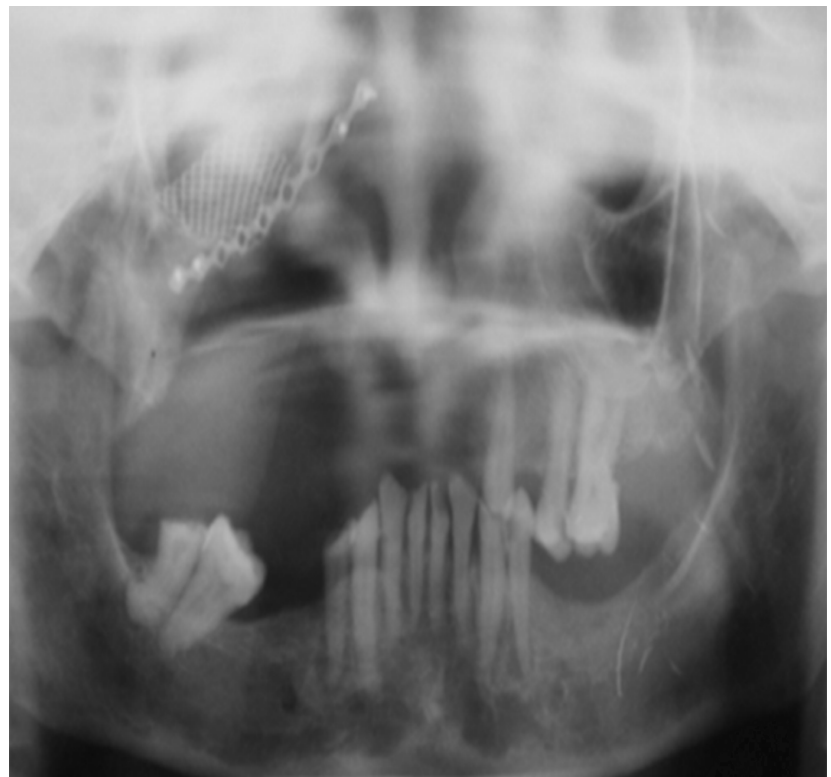

Fig. 6. Ortopantomografía post-operatorio inmediato, donde se evidencia el material de reconstrucción.

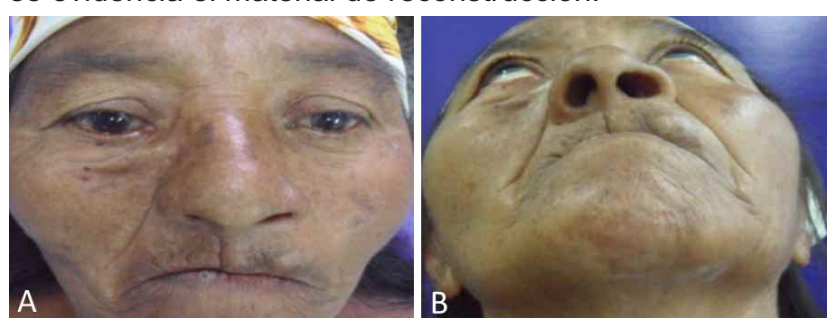

Fig. 7. A. Vista frontal y B. Vista inferior, un mes de postoperatorio, con cicatrización cutánea imperceptible y sin atrapamiento ocular. removidos. Se colocó una placa de reconstrucción de 1,5 (Fig. 5B) la cual se adaptó y fijó mediante cuatro tornillos monocorticales, así como una malla de titanio en el piso de órbita para el soporte del globo ocular.

Se ejecutó la restauración con puntos fijos para evitar la distorsión por pérdida de apoyo esquelético debido a la resección del tumor, así como por la disección del colgajo de sus orígenes óseos. Finalmente se realizó la síntesis quirúrgica por planos sub-cutáneos e intraoral con sutura de Nylon 5-0 y reabsorbible 3-0 respectivamente.

Macroscópicamente, la muestra tomada midió $6,5 \mathrm{~cm} \times 6 \mathrm{~cm} \times 5 \mathrm{~cm}$ (Fig. $5 \mathrm{C}$ ) y fue enviada nuevamente a estudio histopatológico, el cual resultó compatible con el resultado previo. El control radiográfico a las 24 horas mostro la placa de reconstrucción en posición (Fig. 6), un mes posterior a la cirugía la paciente muestra una cicatrización cutánea favorable (Fig. 7A,B). Los controles postoperatorios a los seis y 12 meses, revelaron la cicatrización de la herida en los tejidos blandos, con adecuada función y simetría facial, sin evidencia de recidiva.

\section{DISCUSIÓN}

De acuerdo a la literatura, el FM representa del 2,3 al $17,7 \%$ de todos los tumores odontogénicos, con una mayor frecuencia en mujeres (Reddy et al.; Kant et al.). EI FM tiende a desarrollarse en adultos jóvenes entre los 25-30 años de edad, con un pico de incidencia de 28 años, siendo rara su aparición en individuos menores de 10 años o mayores de 50 (Meleti et al.; Haser et al., 2016). Con relación a su localización, existe una ligera predilección por presentarse en la región posterior de la mandíbula, y es poco frecuente la afectación de la región anterior o la maxila (Haser et al.; Zayet et al.) en donde tiene un comportamiento más agresivo, pudiendo involucrar el hueso cigomático, seno maxilar e inclusive la órbita (Gupta et al.; Kant et al.; Reddy et al.). En el caso reportado la paciente tenía 52 años al momento de la consulta y la lesión se encontraba en la maxila, lo que es una presentación inusual.

Se cree que la lesión se deriva de la vaina nerviosa y es el resultado de la degeneración de fibromas, lipomas, debido a irritación crónica y el proceso degenerativo que sigue a la anoxemia tisular. Por otra parte, se ha indicado que los mixomas/fibromixomas se desarrollan del tejido mesenquimal del folículo den- 
tario, y por ello son descritos como odontogénicos, sin embargo esta explicación falla en describir los mixomas tisulares. EI FM probablemente se genere de las estructuras de soporte del diente como la encía y el ligamento periodontal (Dietrich et al.; Cordero Carrasco et al.; Gupta et al.; Kant et al.)

En cuanto a su comportamiento clínico, los FM son lesiones localmente invasivas y agresivas, pudiendo comprometer las estructuras anatómicas vecinas (Gandhiraj et al.; Gupta et al.; Reddy et al), lo cual fue evidenciado en la paciente de este caso, en la cual el tumor invadió el seno maxilar, pared lateral de la fosa nasal y piso de órbita respectivo.

Debido a que el FM suele ser asintomático en sus fases iniciales (Infante-Cossío et al.; Kant et al.; Reddy et al.), es difícil que el paciente logre identificarlo o acuda tempranamente a recibir la atención adecuada, aumentando la probabilidad que sea tratado cuando el tumor esté en una fase avanzada, como lo fue en el caso presentado. Imagenológicamente, los primeros hallazgos son observados en radiografías odontológicas de rutina, como imágenes bien delimitadas uni o multiloculares en patrón de pompas de jabón, raqueta de tenis o panal de abejas (Dietrich et al.; Cordero Carrasco et al.; Gandhiraj et al.; Gupta et al.; Kant et al.; Meleti et al.). El empleo de la TC permite identificar la expansión de tablas óseas, invasión del seno maxilar, fosas nasales, el piso de órbita o celdillas etmoidales (Gupta et al.; Kant et al.; Reddy et al.). En el caso reportado la imagen presentaba un aspecto unilocular, radiopaca y de márgenes mal definidos, probablemente debido a su localización en la maxila, siendo que la TC posibilito evaluar con precisión la localización de la lesión y su relación con las estructuras vecinas.

Considerando las características clínicas, radiológicas y quirúrgicas, el $\mathrm{FM}$ debe distinguirse de condiciones patológicas como el ameloblastoma, el hemangioma central, displasia fibrosa, quistes odontogenicos, quiste aneurismático, granuloma central de células gigantes, metástasis de neoplasias, liposarcoma bien diferenciado y otras entidades poco comunes como el fibroma desmoplásico (Dietrich et al.; Cordero Carrasco et al.; Gupta et al.; Kumar et al.).

La escisión local del FM puede resultar insuficiente por su naturaleza infiltrativa, sin embargo, esta ha sido la terapéutica seleccionada cuando el paciente es muy joven (Gandhiraj et al.; Meleti et al.; Omeje et al.). Algunos autores señalan que la extirpación quirúrgica con márgenes de seguridad es el procedimiento indicado (Haser et al.; Infante-Cossío et al.; Gupta et al.). En cuanto a la posibilidad de reconstrucción inmediata, se ha reportado el uso de injerto de cresta iliaca (Infante-Cossío et al.) o fíbula (Haser et al.). En el presenta caso se realizó un abordaje extraoral Weber-Fergusson, el cual permitió exponer la lesión completa y su observación directa, buscando la extirpación total de FM y reducir la posibilidad de recidiva. La incisión del abordaje puede ser realizada en partes o extenderse si es necesario mediante incisiones adicionales y las disecciones correspondientes, asimismo, la cicatriz externa es mínima debido a que se localiza en las subunidades estéticas de la cara (Assis et al., 2014).

El abordaje Weber-Ferguson comienza con una incisión vertical la cual es realizada a través del labio superior en el filtrum desde el surco nasolabial hasta el margen bermellón, el extremo inferior se extiende hasta la línea media y luego verticalmente en la línea media a través de la mucosa vestibular al surco gingival vestibular, de manera que el labio superior es completamente dividido y deben ligarse las arterias labiales. La incisión cutánea externa se extiende transversalmente desde el extremo superior de la incisión labial en el surco nasolabial más allá del ala nasal y después superiormente a lo largo del surco hasta el parpado inferior. La incisión nasal lateral debe ser elevada en la unión de la subunidad nasal (Rajasekhar et al., 2012; Bhattacharjee et al., 2018).

Durante la cirugía deben considerarse algunos factores para alcanzar un resultado estético favorable y minimizar la posibilidad de una comunicación bucosinusal. En este sentido, se sugiere que la incisión se mantenga a 1-2 mm lateral a la base de la nariz, lo que facilita la sutura durante el cierre. Asimismo, la incisión nasal lateral debe ejecutarse a lo largo del margen topográfico entre el carrillo y la nariz para mejorar la estética. Por su parte, la incisión intraoral, puede realizarse a lo largo del margen alveolar, para lo cual existen dos abordajes distintos, uno en la mucosa vestibular y el otro en los márgenes cervicales de los dietes presentes (Assis et al.), en el presente caso se realizó en el surco vestibular extendiéndose hacia la línea media del paladar duro. Por último, es imperativa la necesidad de adhesión de los tejidos blandos a la estructura esquelética, debido a que los problemas de suspensión pueden conducir a ectropión, aumento de cicatrices o un tercio medio facial colgante (Assis et al.). 
El tiempo quirúrgico reconstructivo de defectos óseos post remoción de lesiones de gran tamaño y de límites imprecisos, opcionalmente suelen realizarse posterior al acto quirúrgico inicial. Aunque la lesión de este caso comprometía varias estructuras faciales, siendo localmente invasiva y con un comportamiento agresivo, se decidió realizar la reconstrucción inmediata mediante la colocación de una placa de reconstrucción y malla de titanio para devolverle al paciente funcionalidad y estética del tercio medio de la cara y de soporte para el globo ocular. El pronóstico de la lesión es favorable siempre y cuando se realice el tratamiento adecuado, a un año de la intervención, la paciente no muestra signos o síntomas de recidiva.

GUILLEN, R. G. J.; ORTEGA-PERTUZ, A. I. \& ESPINA, S. M. L. Aggressive odontogenic fibromixoma in maxilla: Surgical approach. Int. J. Odontostomat., 13(1):58-63, 2019.

ABSTRACT: Odontogenic fibromyxoma (FM) is an infrequent benign lesion that shows with invasive characteristics. Considered a variant of the odontogenic myxoma, it presents a controversial mesenchymal origin and is somewhat more frequent in women. The lesion is associated with areas with adjacent dental structures, with its most frequent location in the posterior mandibular region. The objective of this study was to show an aggressive case of FM in a female, located in the posterior area of the maxilla, emphasizing its surgical approach. A case of a 52-year-old woman, who came to the clinic because of a painless, firm mass on the right side of the maxilla. Radiographically, it was seen as a radiopaque image, with poorly defined margins extending in the ipsilateral maxillary sinus; computed tomography showed the expansion of the walls of the antrum and the nasal cavity, with zygomatic compromise and the orbital floor. An incisional biopsy was performed and the histopathological diagnosis was FM. The treatment of choice consisted of block resection, using a WeberFerguson approach, along with reconstruction with a titanium plaque and mesh to guarantee suspension of the eyeball; a new histopathological study, confirmed the initial diagnosis. One year after the surgical procedure, there is no recurrence, and the reconstruction material maintains its position, providing aesthetic and functionality to the patient.

KEY WORDS: odontogenic tumor, myxoma, fibromyxoma, maxilla.

\section{REFERENCIAS BIBLIOGRÁFICAS}

Assis, G. H.; Campos, G. B. P.; Pinto, L. P.; Da Silva, J. S. P. \& Germano, A. R. Atypical presentation of a cementoblastoma treated through a Weber-Ferguson approach. Rev. Odonto Cienc., 29(3):96-100, 2014.

Bhattacharjee, A.; Rudresh, K. B.; Prashanth, R.; Khatua, A. \& Kumar, $\checkmark$. Modified Weber Ferguson incision - A rare traumatic serendipity. Int. J. Contemp. Dent. Med. Rev., 2018:020418, 2018. Disponible en: http://www.ijcdmr.com/index.php/ijcdmr/article/ download/293/265

Cordero Carrasco, E.; Ventura, P. S. \& Carrasco Soto, R. Fibromixoma odontogénico en el maxilar superior: reporte de caso clínico y revisión de literatura. Acta Odontol. Venez., 51(2), 2013. Disponible en: http://www.actaodontologica.com/ediciones/ 2013/2/art-11/

Dietrich, E. M.; Papaemmanouil, S.; Koloutsos, G.; Antoniades, H. \& Antoniades, K. Odontogenic fibromyxoma of the maxilla: a case report and review of the literature. Case Rep. Med., 2011:238712, 2011.

Gandhiraj, S. Odontogenic fibromyxoma of maxilla , managed conservatively-a rare case report. IOSR J. Dent. Med. Sci., 4(5):15-8, 2013

Gupta, I.; Keluskar, V. \& Shetti, A. Odontogenic fibromyxoma of left maxilla - A case report. J. Int. Oral Health, 2(4):79-85, 2010.

Haser, G. C.; Su, H. K.; Hernandez-Prera, J. C.; Khorsandi, A. S.; Wang, B. Y. \& Urken, M. L. Pediatric odontogenic fibromyxoma of the mandible: Case report and review of the literature. Head Neck, 38(1):E25-8, 2016.

Infante-Cossío, P.; Martínez-de-Fuentes, R.; García-Perla-García, A.; Jiménez-Castellanos, E. \& Gómez-Izquierdo, L. Myxofibroma of the maxilla. Reconstruction with iliac crest graft and dental implants after tumor resection. Med. Oral Patol. Oral Cir. Bucal, 16(4):e532-6, 2011.

Kant, C.; Prajapati, V. K.; Singh, V. \& Tiwari, K. M. Odontogenic fibromyxoma of maxilla: a case report. Clin. Rhinol. Int. J., 8(2):848, 2015.

Kumar, M.; Suresh, K. V.; Pramod, R. C.; Baad, R.; Anand, S. R. \& Raghavendra, M. N. An unusual case of odontogenic fibromyxoma of anterior maxilla. Int. J. Maxillofac. Pathol., 3(4):60-3, 2012.

Meleti, M.; Giovannacci, I.; Corradi, D.; Manfredi, M.; Merigo, E.; Bonanini, M. \& Vescovi, P. Odontogenic myxofibroma: a concise review of the literature with emphasis on the surgical approach. Med. Oral Patol. Oral Cir. Bucal, 20(1):e1-6, 2015.

Omeje, K. U.; Amole, I. O.; Osunde, O. D. \& Efunkoya, A. A. Management of odontogenic fibromyxoma in pediatric nigerian patients: a review of 8 cases. Ann. Med. Health Sci. Res., 5(6):461-5, 2015.

Rajasekhar, G.; Vura, N. G.; Sudhir, R.; Dhanala, S. \& Alwala, A. M. Versatility of Dieffenbach's modification of Weber Fergusson's approach for treatment of maxillary pathologies. J. Maxillofac. Oral Surg., 11(4):416-9, 2012

Reddy, G. S.; Kumar, B. S.; Muppa, R.; Regonda, S. K. \& Tvs, H. K. Odontogenic fibromyxoma of maxilla: a rare case report. Case Rep. Dent., 2013:345479, 2013.

Zayet, M. K. \& Eiid, S. B. Multiple fibromyxomas of the jaws: A case report. Imaging Sci. Dent., 44(3):237-41, 2014.

Dirección para correspondencia:

Dra. Ana Isabel Ortega-Pertuz

Instituto de Investigaciones

Facultad de Odontología

Universidad del Zulia

Maracaibo

Estado Zulia - VENEZUELA

Email: anitaortegav@gmail.com

Recibido : 30-08-2018

Aceptado: 02-01-2019 